\title{
El valor jurídico y práctico del proyecto de artículos de la CDI sobre el derecho \\ de los acuíferos transfronterizos
}

Fecha de recepción: 28 de enero de 2019

Fecha de aceptación: 27 de julio de 2019

Doi: https://doi.org/10.12804/revistas.urosario.edu.co/acdi/a.7610

\section{Laura Movilla Pateiro* \\ Francesco Sindico ${ }^{* *}$}

Resumen: este trabajo aborda el valor jurídico y práctico que puede llegar a tener el proyecto de artículos sobre el derecho de los acuíferos transfronterizos de la CDI, sobre el cual no parece previsible la adopción de una convención a corto plazo. Para ello, se toma como referencia el estudio del valor jurídico y práctico que han tenido otros trabajos de la CDI que no han llegado a convertirse en una convención internacional en vigor. Este análisis permite apreciar que su aplicación práctica depende no tanto

* Profesora, doctora de derecho internacional público y relaciones internacionales en la Universidade de Vigo (España). Licenciada en derecho por la Universidade de Vigo (España), máster en estudios internacionales por la Universidad del País Vasco (España), doctora en derecho por la Universidade de Vigo (España) e investigadora postdoctoral en el Strathclyde Centre for Environmental Law and Governance (Glasgow, Reino Unido). ORCID: https://orcid.org/0000-0001-6411-9957Correo electrónico: lauramovilla@uvigo.es ** Codirector del Strathclyde Centre for Environmental Law and Governance. Profesor de Derecho Internacional del Medio Ambiente, Universidad de Strathclyde, Glasgow, Escocia, Reino Unido. Doctor en derecho por la Universidad Jaume I de Castellón de la Plana (España). Correo electrónico: francesco.sindico@strath.ac.uk. ORCID: https:// orcid.org/0000-0001-9320-0762?lang=en

Para citar este artículo: Movilla Pateiro, L. \& Sindico, F. "El valor jurídico y práctico del proyecto de artículos de la CDI sobre el derecho de los acuíferos transfronterizos". Anuario Colombiano de Derecho Internacional (ACDI) 13, 2020, 105-132. https://doi.org/10.12804/revistas.urosario.edu.co/acdi/a.7610 
de su forma, sino más bien del valor jurídico de su contenido, es decir, de su naturaleza consuetudinaria o de desarrollo progresivo. En el último caso, de su capacidad para generar nuevas costumbres internacionales a través de su aceptación por los Estados.

Palabras clave: acuíferos transfronterizos, Comisión de Derecho Internacional, codificación, desarrollo progresivo, proyecto de artículos.

The Normative and Practical Value of the ILC's Draft Articles on the Law of Transboundary Aquifers

Abstract: This paper addresses the normativity of the Draft Articles on the Law of Transboundary Aquifers, which are not likely to become an international treaty in the short term. In order to address this issue, the paper generally focuses on the work of the United Nations International Law Commission that has not led to an international convention. The comparative analysis undertaken leads to the conclusion that the practical application of the Draft Articles on the Law of Transboundary Aquifers depends less on its final format and more on the normativity of its content. In other words, what will make them resonate in international practice is the extent to which provisions therein reflect customary international law or represent progressive development in it. It will also be interesting to see the extent to which future application of the Draft Articles on the Law of Transboundary Aquifers themselves may lead to the development of new international customary law in this field.

Keywords: Transboundary aquifers, International Law Commission, codification, progressive development, draft articles.

\section{O valor jurídico e prático do projeto de artigos da CDI sobre o direito dos aquíferos transfronteiriços}

Resumo: este trabalho aborda o valor jurídico e prático que pode chegar a ter o projeto de artigos sobre o direito dos aquíferos transfronteiriços da $\mathrm{CDI}$, sobre o qual não parece previsível que se vá a adotar uma convenção a curto prazo. Para isto, toma-se como referência o estudo do valor jurídico e prático que têm tido outros trabalhos da CDI que não têm chegado 
a se converter em uma convenção internacional em vigor. Esta análise nos permite apreciar que sua aplicação prática depende não tanto de sua forma, mas do valor jurídico de seu conteúdo, é dizer, de sua natureza consuetudinária ou de desenvolvimento progressivo; e, neste último caso, de sua capacidade para gerar novas costumes internacionais através de sua aceitação pelos Estados.

Palavras-chave: aquíferos transfronteiriços, Comissão de Direito Internacional, codificação, desenvolvimento progressivo, projeto de artigos.

\section{Introducción}

Los acuíferos transfronterizos son un recurso natural de extrema relevancia. En un contexto mundial de crisis hídrica y de aumento de los impactos del cambio climático, se estima que las aguas subterráneas representan alrededor del $97 \%$ del agua dulce del planeta ${ }^{1}$ y son fundamentales para el abastecimiento de agua de la población mundial y de los ecosistemas. ${ }^{2}$ La mayor parte de estas aguas se encuentran en acuíferos, es decir, en formaciones geológicas permeables capaces de almacenar, transmitir y proporcionar cantidades aprovechables de agua, ${ }^{3}$ las cuales son consideradas transfronterizas cuando partes de las mismas se encuentran situadas en el territorio de dos o más Estados. ${ }^{4}$ El último inventario realizado calcula en casi 600 el número de acuíferos y masas de agua subterráneas transfronterizas del planeta. ${ }^{5}$

Sin embargo, su regulación a nivel internacional se encuentra todavía en un estado incipiente, razón por la cual la Comisión de Derecho Internacional (CDI) de las Naciones Unidas comenzó los trabajos sobre

\footnotetext{
1 World Water Assessment Programme, Water, a shared responsibility. The United Nations world water development report 2006, Unesco, 121.

2 Ibíd., p.13.

3 Artículo 2. a) del Proyecto de artículos sobre el derecho de los acuíferos transfronterizos, anexado a la Resolución 63/124 de la Asamblea General de 11 de diciembre de 2008, A/RES/63/124.

4 OMm/Unesco, Glosario Hidrológico Internacional, OMM-N $385,2012,18$.

5 IGRAC, Transboundary aquifers of the world. Special edition for the 7 th World Water Forum 2015, 2015.
} 
este tema en 2002, fruto de los cuales adoptó en 2008 un proyecto de 19 artículos, de los que la Asamblea General tomó nota y anexó a una resolución, alentando a que los Estados la tengan en cuenta en sus disposiciones cuando celebren arreglos bilaterales o regionales sobre este recurso.

A pesar de no ser una convención internacional en vigor, este proyecto puede tener una importante aplicación internacional. Por ello, se abordará en este trabajo su posible valor jurídico y práctico, tanto desde el punto de vista formal — como proyecto de artículos de la CDI—, como material, atendiendo al carácter de desarrollo progresivo o derecho consuetudinario de su contenido. Para ello, este estudio se estructura en tres partes. En la primera se hará una introducción a la labor que realiza la CDI y los efectos jurídicos que han desplegado otros proyectos de artículos de la CDI que tampoco han llegado a ser convenciones internacionales pero que, no obstante, han sido aplicados por los Estados, tribunales u otras instancias internacionales. Se prestará especial atención a los relativos a la responsabilidad internacional del Estado, que servirán de referente para el análisis posterior del trabajo de la CDI sobre los acuíferos transfronterizos. En la segunda parte se ofrecerá un breve recorrido por la gestación en la CDI del proyecto de artículos sobre el derecho de los acuíferos transfronterizos y las sucesivas discusiones sobre este en el seno de la Asamblea General. En la tercera parte, se presentarán ejemplos concretos de la práctica internacional en los que se ha recurrido a este proyecto de artículos, sobre la base del análisis realizado en los apartados anteriores, para evaluar su potencial valor normativo y práctico, a sabiendas que muy probablemente no va a convertirse en un tratado internacional, al menos no en breve.

\section{Los proyectos de artículos de la CDI y sus efectos jurídicos y prácticos}

La CDI fue creada en 1947 por la Asamblea General como órgano subsidiario encargado de la codificación y desarrollo progresivo del derecho internacional. A este respecto, el artículo 15 de su Estatuto se refiere a la distinción entre codificación y desarrollo progresivo, cuya iniciativa se atribuyó inicialmente a la CDI y a la Asamblea General, y estableció originariamente dos procedimientos distintos para una y otra labor. ${ }^{6}$ Sin embargo,

6 Véanse los artículos 16 y 17 y 18 a 23 del Estatuto de la CDI. 
esa distinción no se ha podido mantener en la práctica y todos los procedimientos incluyen, inevitablemente, aunque en distinta medida según la materia de la que se trate, tanto codificación como desarrollo progresivo. Por ello, suele hacerse referencia al trabajo de la CDI como "codificación" en un sentido amplio, es decir, incluye tanto labores de codificación en sentido estricto, como de desarrollo progresivo. En esa codificación se combinan los trabajos de estudio de la CDI — principalmente a través de los relatores especiales designados en cada caso- los debates en la Sexta Comisión de las Naciones Unidas y las observaciones y comentarios de los gobiernos.

\subsection{Evolución de la labor codificadora de la CDI}

El resultado de los trabajos de la CDI ha sido tradicionalmente un proyecto de artículos sobre el que los Estados deciden lanzar un proceso de negociación intergubernamental dirigido a alcanzar un tratado internacional. ${ }^{7}$ Sin embargo, también pueden dar lugar, y cada vez más, a otros resultados ${ }^{8}$ que, sin ser jurídicamente vinculantes, pueden llegar a tener una importante aplicación en la práctica internacional y se enmarcan de forma más amplia en el creciente fenómeno de "desformalización" del derecho internacional. Este alejamiento del planteamiento original de los trabajos de la CDI se debe a una conjunción de razones, entre las que se encuentran las siguientes: la propia elección de las materias de trabajo, muy controvertidas o politizadas en algunos casos, ${ }^{10}$ sobre todo, una vez agotados los grandes temas sobre los que existía un consenso

\footnotetext{
7 De este modo, los trabajos de la CDI han dado lugar a la negociación, adopción y entrada en vigor de convenciones tan relevantes como las de Viena sobre relaciones diplomáticas (1961) y consulares (1963), las cuatro convenciones de Ginebra sobre el derecho del mar de 1958, la Convención de Viena sobre el Derecho de los Tratados de 1969 y la Convención sobre las Inmunidades Jurisdiccionales de los Estados y de sus Bienes de 2004.

8 Véase el artículo 23 del Estatuto de la CDI.

9 Véanse Jean D'Aspremont, "The Politics of Deformalization in International Law", Goettingen Journal of International Law 3, 2 (2011): 503-550; Jean D'Aspremont, Formalism and the sources of International Law. A Theory of the Ascertainment of Legal Rules, (Oxford University Press, 2011).

10 Es el caso, por ejemplo, del fracasado Proyecto de Código de Crímenes contra la Paz y la Seguridad de la Humanidad.
} 
más o menos generalizado; el riesgo de adoptar convenciones que tarden mucho tiempo en entrar en vigor o que no lleguen a hacerlo; ${ }^{11}$ la intención de buscar una mayor aceptación de los textos resultantes, o la necesidad de fórmulas flexibles que se adapten mejor a las necesidades de la sociedad internacional y a la complejidad de los temas que se puedan tratar. Ello ha motivado que la Asamblea General, por un lado, posponga repetidamente la decisión sobre la acción apropiada después de que la CDI le haya presentado el proyecto de artículos. Por otro lado, a llevado a que la propia CDI favorezca, cada vez de forma más habitual, aunque no exclusivamente, métodos de trabajo que conducen directamente a la elaboración de instrumentos no vinculantes. ${ }^{12}$ De este modo, cada vez resulta más frecuente que la intención sea la de hacer directamente un estudio o establecer directrices, principios o conclusiones para que los Estados los tengan en cuenta en su práctica internacional. ${ }^{13}$

11 Por ejemplo, el otro gran trabajo de la CDI sobre recursos hídricos, sobre cuya base se elaboró la Convención sobre el Derecho de los Usos de los Cursos de Agua Internacionales de 1997, necesitó 17 años para conseguir el número de ratificaciones necesarias (35) para entrar en vigor.

12 Véanse Santiago Villalpando, "Codification Light: A New Trend in the Codification of International Law at the United Nations", Anuário Brasileiro de Direito Internacional VIII, no. 2 (2013): 117-155; Fernando Lusa Bordin, "Reflections of customary international law: the authority of codification conventions and ILC draft articles in International Law", International and Comparative Law Quarterly 63, no. 3 (2014): 536.

13 Se pueden citar, entre otros, los célebres informe y conclusiones del Grupo de Estudio sobre la Fragmentación del Derecho Internacional presentados en 2006. Como ejemplos de trabajos destinados a guiar la práctica de los Estados — sin la adopción de una convención - están, entre otros, el Proyecto de Principios sobre la Asignación de la Pérdida Causada por Daños Transfronterizos Resultantes de Actividades Peligrosas de 2007 (véase, infra, sección 1.2.2), la Guía de la práctica sobre las reservas a los tratados aprobada en 2011, las recientes conclusiones sobre la identificación del derecho consuetudinario internacional de 2018 o los trabajos en marcha sobre un proyecto de directrices para la protección de la atmósfera y un proyecto de principios sobre la protección del medio ambiente en relación con los conflictos armados. En este sentido, se ha planteado incluso la posibilidad de que la CDI se dedique exclusivamente a actividades de recomendación o asesoramiento. Véase Ana Gemma López Martín, "La codificación del Derecho Internacional en el umbral del Siglo XXI. Luces y sombras en la labor de la CDI", Anuario Hispano-Luso-Americano de Derecho Internacional 15, (2001): 385. 


\subsection{Valor jurídico y práctico de los proyectos de la CDI que no han dado lugar a convenciones}

De entre la tipología de trabajos de la CDI que no han resultado en la adopción de una convención que haya alcanzado vigencia, se prestará especial atención a los proyectos de artículos de la CDI sobre cuya base la Asamblea General, en muchos casos, propuesto por la propia CDI, ha decidido no negociar una posterior convención. Ese es el caso del proyecto de artículos sobre el derecho de los acuíferos transfronterizos que nos ocupa en este trabajo, pero, también, de otros, como el proyecto de artículos sobre la responsabilidad de los Estados por hechos ilícitos de 2001, o sobre la prevención de los daños resultantes de actividades peligrosas de 2006, los cuales examinaremos en el siguiente apartado como ejemplos paradigmáticos de textos de este tipo que gozan, sin embargo, de una importante aceptación y aplicación práctica.

\subsubsection{E1 Proyecto de artículos de la CDI sobre la responsabilidad de los Estados por hechos ilícitos}

Este proyecto de $2001^{14}$ fue el resultado de un largo y complejo proceso iniciado en los años cincuenta. Las dificultades en su proceso de elaboración se debieron a que la mayoría de sus artículos recogían — como le va a ocurrir también a los relativos al derecho de los acuíferos transfronterizos- desarrollo progresivo en esa materia, más que una codificación en sentido estricto de ella, ${ }^{15}$ por lo que los debates fueron controvertidos y se extendieron duramente varios años. Más allá de su contenido material, ${ }^{16}$ se produjeron también importantes discrepancias en torno a dos cuestiones íntimamente relacionadas entre sí y que se van a plantear posteriormente también en relación con el derecho de los acuíferos transfronterizos: si debía establecerse un mecanismo de solución de controversias y la forma final que debía adoptar dicho proyecto de artículos.

14 Documentos Oficiales de la Asamblea General, quincuagésimo sexto periodo de sesiones, Suplemento no. 10, A/56/10, párrs. 76-77.

15 David D. Caron, "The ILC Articles on State Responsibility: The Paradoxical Relationship between Form and Authority," The American Journal of International Law 96, (2002), p. 854.

16 Véase al respecto, entre otros, James Crawford, The International Law Commission's Articles on State Responsibility: Introduction, Text, and Commentaries, (Cambridge University Press, 2002). 
En relación con la resolución de controversias, aunque se había aprobado en primera lectura una parte III que incluía disposiciones detalladas al respecto, ${ }^{17}$ esta fue finalmente descartada ante las discrepancias que había desatado. Sin embargo, la CDI, en su informe a la Asamblea General, llamó su atención a ese mecanismo de solución de controversias y dejó que fuese ella quien examinara si debían incluirse y en qué forma esas disposiciones, en el caso de que decidiera elaborar una convención. ${ }^{18}$

No obstante, la intención de elaborar una convención sobre la responsabilidad de los Estados por hechos ilícitos se diluyó poco a poco. ${ }^{19}$ Los principales argumentos a favor insistían en la extrema relevancia del tema de la responsabilidad internacional en el derecho internacional y la certidumbre, fiabilidad y fuerza vinculantes adicionales que daría un tratado a esas normas para ayudar a su consolidación, ${ }^{20}$ así como en el importante papel que desempeñan incluso las convenciones no ratificadas. ${ }^{21}$

A la inversa, entre las razones en contra de la adopción de una convención internacional se señalaron: la dificultad de declarar los elementos básicos del derecho internacional en una convención; el temor a una "codificación inversa", es decir, a que normas consuetudinarias que se incluyesen en una convención dejasen de tener tal condición si esta no fuese ratificada, o la posibilidad de reservas o falta de cooperación por parte de los Estados. ${ }^{22}$ Además, y prediciendo acertadamente cual sería el futuro de ese proyecto de artículos, se argumentó también que, si fuese aprobado por una resolución de la Asamblea General, sería considerado como un estudio autorizado de las normas, la práctica y la doctrina, que serviría de orientación a los Estados en este ámbito y contribuiría a la certidumbre jurídica de este. ${ }^{23} \mathrm{Al}$ contrario, los opositores a un instrumento no vinculante argumentaron que tal forma reduciría la importancia de la

\footnotetext{
17 Document A/CN.4/L.513. State Responsibility. Titles and Texts of Articles Adopted by the Drafting Committee: Part Three and Annex - Reproduced in Document A/CN.4/ SR.2417, para.1

18 Documentos oficiales de la Asamblea General, quincuagésimo sexto periodo de sesiones, Suplemento no. 10, A/56/10, párr. 60.

19 Ibíd., párr. 61.

20 Ibíd., párr. 62.

21 Ibíd., párr. 62.

22 Ibíd., párr. 63.

23 Ibíd. párrs. 63 y 64.
} 
cuestión de la responsabilidad de los Estados en derecho internacional, generaría dudas sobre el valor del texto y no podría impedirse que los Estados añadiesen declaraciones interpretativas a ese instrumento. ${ }^{24}$

En cuanto a la relación entre la forma y el contenido, durante estos debates hubo tanto partidarios de que a) si el proyecto de artículos contuviese una buena dosis de desarrollo progresivo, sería necesaria una convención multilateral; b) a la inversa, precisamente por ello, era necesario un enfoque más cauteloso que no llevase a la elaboración de un instrumento vinculante, y c) el proyecto de artículos combinaba tanto codificación como desarrollo progresivo y que, en todo caso, a la larga, lo relevante no era tanto si se trataba de una u otra cuestión, sino de si sus disposiciones eran lógicas y equilibradas. ${ }^{25}$

Finalmente, ante la división de opiniones sobre cuál debía ser su forma final, la CDI decidió adoptar un enfoque de dos fases. En primer lugar, recomendó que la Asamblea General tomase nota y anexase el proyecto de artículos a una resolución. La segunda fase, teniendo en cuenta la importancia de la materia, implica una posterior consideración de la adopción de una convención en una futura sesión de la Asamblea General, tras haberse comprobado el grado de aceptación por parte de los Estados, en la que se consideraría también la inclusión de provisiones sobre resolución de conflictos. ${ }^{26}$

Esa consideración del tema por parte de la Asamblea General se ha venido realizando hasta la actualidad, retomándose y posponiéndose periódicamente en su agenda de trabajo. Así, en la Resolución 56/83 de 2001, ${ }^{27}$ la Asamblea General tomó nota de estos artículos y los señaló a la atención de los gobiernos "sin perjuicio de la cuestión de su futura aprobación o de otro tipo de medida, según corresponda" y decidió incluir este tema en el programa de su quincuagésimo noveno periodo de sesiones (2004). ${ }^{28}$

\footnotetext{
24 Ibíd., párr. 65.

25 Ibíd., párr. 66.

26 Informe de la Comisión de Derecho Internacional sobre la labor realizada en su quincuagésimo tercer periodo de sesiones, 18 de enero de 2002, A/RES/56/82, párr. 67.

27 Resolución 56/83 de la Asamblea General de 12 de diciembre de 2001, A/RES/53/83.

28 Véase la Resolución 59/35 de la Asamblea General del 2 de diciembre de 2004, A/ RES/59/35.
} 
Desde entonces, se ha examinado también en cuatro ocasiones, ${ }^{29}$ llamándose de nuevo a la atención de los gobiernos, y se ha incluido en la agenda provisional del septuagésimo cuarto periodo de sesiones (2019). Además, desde su Resolución 59/35 de 2004, la Asamblea General está pidiendo al Secretario General que invite a los gobiernos a presentar sus observaciones por escrito sobre medidas futuras, así como que prepare compilaciones de las decisiones de las cortes, tribunales y otros órganos internacionales relativas a los artículos. ${ }^{30}$ En 2008 se creó también un grupo de trabajo en la Sexta Comisión para examinar la cuestión de una convención o la adopción de otro tipo de medida sobre la base de los artículos. ${ }^{31}$ En 2010, la Asamblea General reconoció la importancia de los artículos ${ }^{32}$ y en 2013 y 2016 que hay cada vez más decisiones de cortes, tribunales y otros órganos internacionales que se refieren a los mismos. ${ }^{33}$

\subsubsection{Los trabajos de la CDI sobre los daños transfronterizos derivados de actividades peligrosas}

En 1978, la CDI comenzó a trabajar, de forma paralela, sobre la responsabilidad internacional por las consecuencias perjudiciales de actos no prohibidos por el derecho internacional. Años más tarde, tras varias recomendaciones de los distintos grupos de trabajo establecidos hasta ese momento, la CDI decidió en 1997 subdividir el tema en dos: la prevención de actividades peligrosas y la asignación de la pérdida en caso de producirse dicho daño. ${ }^{34}$

Fruto de los trabajos centrados en la prevención, la CDI adoptó un proyecto de artículos en 2001, que sometió a la Asamblea General con la

29 Resoluciones de la Asamblea General 62/61 del 6 de diciembre de 2007, A/RES/62/61; 65/19 del 6 de diciembre de 2010, A/RES/65/19; 68/104 del 16 de diciembre de 2013, A/RES/68/104, y 71/133 del 13 de diciembre de 2016, A/RES/71/133.

30 Véase, infra, sección 1.2.3.

31 Resolución de la Asamblea General 62/61..., punto 4.

32 Resolución de la Asamblea General 65/19..., punto 1.

33 Resoluciones de la Asamblea General 68/104 y 71/133..., puntos 1 y 2.

34 Documentos Oficiales de la Asamblea General, quincuagésimo segundo período de sesiones, suplemento no.10, A/52/10, párr. 168, y Resolución 52/156 de la Asamblea General del 15 de febrero de 1997, párr. 7. 
recomendación de que elaborase una convención. ${ }^{35}$ En consonancia con esa recomendación, este proyecto incluyó una cláusula relativa a la solución de conflictos en caso de controversias sobre su interpretación o aplicación. Sin embargo, la Asamblea General se limitó a expresar su reconocimiento por la valiosa labor realizada y pidió a la Comisión que reanudase su examen de los temas relacionados con la responsabilidad internacional. ${ }^{36}$

A su vez, los resultados de los trabajos sobre la asignación de la pérdida causada por daños transfronterizos resultantes de actividades peligrosas se recogieron en un proyecto de principios en $2006 .{ }^{37}$ En este caso, la CDI recomendó a la Asamblea General que hiciese suyo el proyecto de principios en una resolución y que instase a los gobiernos a adoptar disposiciones tanto en el ámbito interno como internacional para llevarlos a efecto. ${ }^{38}$ Con posterioridad, ambas cuestiones han sido tratadas de nuevo en cuatro ocasiones, ${ }^{39}$ incluidas en el programa provisional del septuagésimo cuarto periodo de sesiones (2019).

En todas esas ocasiones se ha señalado a la atención de los gobiernos tanto los artículos como los principios. Además, se les ha invitado a presentar observaciones sobre las medidas que puedan adoptarse, especialmente sobre su forma final, teniendo presentes las recomendaciones formuladas por la Comisión al respecto, en particular, sobre la elaboración

35 Documentos oficiales de la Asamblea General, quincuagésimo sexto periodo de sesiones..., párrs. 97-98.

36 Resolución 56/82 de la Asamblea General, de 12 de diciembre de 2001, A/RES/56/82. Informe de la Comisión de Derecho Internacional, quincuagésimo tercer periodo de sesiones...

Véase en detalle sobre el proceso de elaboración de ese proyecto de artículos y su contenido: José Juste Ruiz, "El proyecto de la comisión de derechos internacional sobre prevención del daño transfronterizo resultante de actividades peligrosas", Pacis Artes. Obra homenaje al profesor Julio D. González Campos. Vol. 1: Derecho Internacional Público y Derecho Comunitario y de la Unión Europea, (Edifer, Madrid, 2005), 327-360.

37 Documentos Oficiales de la Asamblea General, sexagésimo primer periodo de sesiones, Suplemento no. 10, A/61/10, párrs. 66-77.

38 Resolución 61/36 de la Asamblea General del 4 de diciembre de 2006, A/RES/61/36. Documentos oficiales de la Asamblea General, sexagésimo primer periodo de sesiones..., párr. 63.

39 Resoluciones 62/68 de la Asamblea General del 6 de diciembre de 2007, A/RES/62/68; 65/28 del 6 de diciembre de 2011, A/RES/65/28; 68/114 del 16 de diciembre de 2013, A/RES/68/114, y 71/143 de la Asamblea General del 13 de diciembre de 2016, A/ $\mathrm{RES} / 71 / 143$. 
de una convención basada en el proyecto de artículos, así como las prácticas relacionadas con su aplicación. Asimismo, la Asamblea General ha solicitado desde el 2011 al Secretario General compilaciones de las decisiones de cortes, tribunales y otros órganos internacionales relativas a los artículos y principios.

\subsubsection{Aplicación práctica de los trabajos de la CDI sobre la responsabilidad internacional del Estado}

Las recopilaciones presentadas hasta ahora por el Secretario General a petición de la Asamblea General sobre los trabajos de la CDI a propósito de la responsabilidad internacional del Estado resultan indicativas de su gran aplicación práctica. Esto es cierto especialmente para el Proyecto de Artículos sobre la Responsabilidad del Estado por Hechos Ilícitos, el cual constituye una muestra de "la relación paradójica entre forma y autoridad", ya que puede que haya tenido incluso más influencia que si hubiese llegado a ser un tratado multilateral. De hecho, desplegó su influencia incluso en la forma de proyecto parcial. ${ }^{40}$

De este modo, las cuatro compilaciones presentadas por el Secretario General a petición de la Asamblea General entre 2007 y $2016^{41}$ muestran cómo se hace referencia a los proyectos de artículos sobre la responsabilidad del Estado por hechos ilícitos en 282 decisiones de una gran heterogeneidad de tribunales y otros órganos internacionales, tanto globales como regionales. A su vez, dos exámenes de $2013^{42}$ y $2016^{43}$ sobre la prevención del daño transfronterizo resultante de actividades peligrosas y la asignación de la pérdida en caso de producirse dicho daño reflejan cómo han sido invocados, al menos, en nueve decisiones judiciales y arbitrales. Como sabemos, la jurisprudencia constituye un

40 Caron, “The ILC Articles...”, p. 858.

41 Responsabilidad del Estado por hechos internacionalmente ilícitos. Compilación de las decisiones de cortes, tribunales y otros órganos internacionales. Informe del Secretario General del 1 de febrero 2007, A/62/62; 30 de abril de 2010, A/65/76; 30 de abril de 2013, A/68/72, y 21 de abril de 2016, A/71/80.

42 Examen de la prevención del daño transfronterizo resultante de actividades peligrosas y asignación de la pérdida en caso de producirse dicho daño. Compilación de las decisiones de las cortes, tribunales y otros órganos internacionales. Informe del Secretario General del 13 de junio de 2013, A/68/94.

43 Ibíd., 23 de junio de 2016, A/71/98. 
medio subsidiario para la determinación de la existencia y contenido del derecho consuetudinario internacional. ${ }^{44}$

Por lo tanto, los tres textos resultantes son ejemplos de trabajos de la CDI que, aunque por distintas razones no han dado lugar a convenciones internacionales, han sido aplicados igualmente en la práctica. Como se ha visto, el Proyecto de Artículos sobre la Responsabilidad de los Estados por Hechos Ilícitos se corresponde con trabajos sobre los que la propia CDI no estimó conveniente utilizarlo de momento como base para una convención. Sobre el Proyecto de Artículos sobre Prevención de Daños Resultantes de Actividades Peligrosas, la CDI recomendó a la Asamblea General la elaboración de un instrumento vinculante y fue ese órgano el que ha pospuesto una decisión sobre su forma final. Por último, el proyecto de principios sobre la asignación de la pérdida ejemplifica un caso de trabajos de la CDI, cuyo planteamiento inicial era la elaboración de un texto no vinculante. En los tres casos, y aunque en diferente grado, se encuentran también trabajos que no contaban con una base consuetudinaria ampliamente consolidada en el momento de su elaboración.

Aunque existe, obviamente, una importante diferencia entre los temas abordados, tanto en términos sustantivos, como en relación con su relevancia general dentro del derecho internacional, el análisis de los trabajos de la CDI sobre la responsabilidad internacional del Estado va a resultar un precedente indispensable en el ejercicio de reflexión sobre el posible valor jurídico y práctico del Proyecto de Artículos sobre el Derecho de los Acuíferos Transfronterizos. En concreto, el texto que va a resultar más práctico es el relativo a la responsabilidad de los Estados por hechos ilícitos, debido a las similitudes tanto en las decisiones sobre su forma tomadas por la CDI y la Asamblea General, como en las causas que llevaron a ellas en ambos casos.

\section{E1 derecho internacional de los acuíferos transfronterizos y el proyecto de artículos de la CDI de 2008}

A pesar de la enorme relevancia cuantitativa y cualitativa que los acuíferos transfronterizos tienen tanto para la humanidad como para el planeta, ${ }^{45}$

\footnotetext{
44 Conclusión 13 de la CDI sobre la identificación del derecho consuetudinario internacional. Resolución 73/203 de la Asamblea General del 20 de diciembre de 2018, A/ RES/73/203.

45 Véase, supra, la introducción a este trabajo.
} 
su regulación resulta todavía muy escasa, sobre todo si se la compara con la de las aguas superficiales. Por una parte, el número de tratados o acuerdos internacionales celebrados entre Estados sobre acuíferos concretos es marcadamente escaso, no alcanza ni a una decena. ${ }^{46}$ Por otra, a nivel global, además de algunas interesantes y pioneras propuestas doctrinales, ${ }^{47}$ existen dos convenciones internacionales que ofrecen un tratamiento limitado de este recurso. La Convención de las Naciones Unidas sobre el Derecho de los Usos de los Cursos de Agua Internacionales para Fines Distintos a la Navegación de 1997, fruto también de los trabajos de la CDI en vigor desde 2014, aunque con un número todavía escaso - 36 Estados parte- incluye a las aguas subterráneas conectadas con las de superficie en su definición de curso de agua internacional. ${ }^{48}$ Sin embargo, esta no desarrolla en el resto de sus disposiciones un régimen jurídico que tenga en cuenta sus características particulares y no resultaría aplicable a la totalidad de los acuíferos transfronterizos. A su vez, el Convenio para la Protección y Utilización de los Cursos de Agua Transfronterizos y de los Lagos Internacionales, celebrado en el marco de la Comisión de las Naciones Unidas para Europa (UNECE) en 1992, en vigor desde 1996 y abierto a la ratificación por cualquier Estado miembro de las Naciones Unidas desde 2013, se refiere en general a las "aguas transfronterizas", incluyendo tanto superficiales como subterráneas. Sin embargo, la referencia a estas últimas no habría generado un efecto práctico en la acción de los Estados ${ }^{49}$ a pesar de haberse desarrollado también en el marco de

46 Véase Laura Movilla Pateiro, "Ad hoc legal mechanisms governing transboundary aquifers: current status and future prospects," Water International 40, 6 (2016): 851-865 y Stefano Burchi, "Legal Frameworks for the Governance of International Transboundary Aquifers: Pre- and Post-ISARM Experience," Journal of Hidrology: Regional Studies 20, (2018): 15-20.

47 Véanse el Proyecto de Tratado Bellagio de 1989 o, en el marco de la ILA, Reglas de Helsinki sobre el uso de las aguas de los ríos internacionales de 1966, las Reglas de Seúl sobre aguas subterráneas internacionales de 1986 y las Reglas de Berlín sobre recursos hídricos de 2004.

48 Artículo 2 de la Convención de Nueva York de 1997.

49 Pilar Carolina Villar, "International Cooperation on Transboundary Aquifers in South America and the Guarani Aquifer Case," Revista Brasileña de Política Internacional 59, no. 1 (2016): 4 . 
la UNECE unas disposiciones modelo sobre aguas subterráneas transfronterizas en $2012 .^{50}$

Este todavía escaso marco jurídico internacional, unido a la gran relevancia de este recurso natural sobre el cual se tiene cada vez más información y se encuentra sobreexplotado ${ }^{51}$ ha dado lugar a la necesidad de profundizar en su regulación. Así, consciente de las limitaciones de la Convención de Nueva York de 1997 a la hora de abordar las aguas subterráneas, la CDI adoptó la Resolución sobre las Aguas Subterráneas Confinadas Transfronterizas al mismo tiempo que adoptó el Proyecto de Artículos de la Convención de Nueva York en 1994 . $^{52}$ Entre otras cuestiones, se reconocía en esa resolución la necesidad de realizar esfuerzos continuados para elaborar normas relativas a dichas aguas. ${ }^{53}$ Años más tarde, la CDI se centró en exclusiva en esa cuestión, que dio lugar a sus trabajos y al Proyecto de Artículos sobre el Derecho de los Acuíferos Transfronterizos en 2008.

\subsection{Los trabajos en la CDI (2002-2008)}

Los trabajos para su elaboración comenzaron en el 2000, cuando la CDI incluyó el tema de los "recursos naturales compartidos entre Estados" en su agenda de trabajo a largo plazo. ${ }^{54}$ Posteriormente, en 2002, decidió incluir efectivamente el tema en su programa de trabajo, nombró a Chusei Yamada como Relator Especial y estableció un grupo de trabajo. ${ }^{55}$ El relator decidió limitar el ámbito del estudio a las aguas subterráneas, el petróleo y el gas natural y adoptar un enfoque por etapas, comenzando con las

50 Draft Model Provisions on Transboundary Groundwaters, ECE/MP.WAT/2012/L.5. Véase UNECE, Model Provisions on Transboundary Groundwaters, (New York and Geneva, United Nations, 2014).

51 Véase Alexandra S. Richey, Brian F. Thomas, Min-Hui Lo, et al., "Quantifying Renewable Groundwater Stress with GRACE”, Water Resources Research 51, no. 7 (2015): 5217-38.

52 Anuario de la Comisión de Derecho Internacional II, segunda parte (1994), 144.

53 Ibíd., párr. 5 de la resolución.

54 Documentos oficiales de la Asamblea General, quincuagésimo quinto periodo de sesiones, Suplemento no. 10, A/55/10, párr. 729.

55 Documentos oficiales de la Asamblea General, quincuagésimo séptimo período de sesiones, Suplemento no. 10 y corrección (A/57/10 y Corr.1), párr. 518. 
primeras. ${ }^{56}$ Los estudios sobre el petróleo y el gas natural fueron posteriormente abandonados ${ }^{57}$ y el objeto del estudio se redirigió a los “acuíferos transfronterizos", reconociendo así la doble dimensión de este recurso, que no está compuesto solamente de las aguas subterráneas propiamente dichas, sino también por la formación geológica que las contiene. ${ }^{58}$

Durante los debates, algunos Estados se mostraron partidarios de que la forma final del texto fuese una convención marco, en cuyo caso, algunos sugirieron que sería necesario tomar precauciones para que no sustituyese acuerdos previos vigentes o limitase la capacidad de los Estados para celebrarlos. Otros eran favorables a la adopción de un instrumento no vinculante, bajo la forma de una resolución de la Asamblea General que contuviese principios generales orientadores para la celebración de acuerdos o en la forma de directrices o un conjunto de principios modelo. Otros consideraban más razonable aplazar la decisión sobre la forma final del texto y alguno, incluso, que la aprobación de un instrumento no vinculante podría constituir el primer paso para el establecimiento de un régimen adecuado para la utilización de todos los recursos naturales compartidos. ${ }^{59}$

Para el Relator Especial el objetivo final debería ser preparar una convención jurídicamente vinculante, ya que consideraba que

el derecho de los acuíferos transfronterizos es el complemento de la Convención sobre el derecho de los usos de los cursos de agua internacionales para fines distintos de la navegación de 1997 y la cuestión de los acuíferos es tan importante o incluso más importante para la Humanidad que las aguas de superficie. ${ }^{60}$

\footnotetext{
56 Recursos naturales compartidos: primer informe sobre el esbozo del tema, 30 de abril de 2003, un Doc. NU. A/CN.4/533, párr. 4.

57 Recursos naturales compartidos: viabilidad del trabajo futuro en materia de petróleo y gas. Documento preparado por el Sr. Shinya Murase, Comisión de Derecho Internacional, sexagésimo segundo periodo de sesiones, A/CN.4/621.

58 Segundo Informe sobre recursos naturales compartidos: aguas subterráneas transfronterizas. Preparado por el Sr. Chusei Yamada, Relator Especial, A/CN.4/539, 9 de marzo de 2004, párr. 2.

59 Véanse los documentos oficiales A/C.6/62/SR.22, A/C.6/62/SR.23, A/C.6/62/ SR.24, A/C.6/SR.25 y A/C.6/62/SR.26.

60 Quinto informe sobre recurso naturales compartidos: acuíferos transfronterizos. Preparado por Chusei Yamada, Relator Especial, 21 de febrero de 2008, A/CN.4/591, párr. 8.
} 
No obstante, reconocía que no era realista pensar que ese objetivo se pudiese alcanzar a corto plazo debido a la falta de consenso en la comunidad internacional y a que el procedimiento de codificación de una convención en la actualidad resulta más largo que en décadas anteriores, aun cuando exista consenso. Además, consciente de la crisis mundial sobre los recursos hídricos, consideró más práctico que se tomasen medidas urgentes y que los Estados afectados concretasen acuerdos bilaterales o regionales sobre la base de las disposiciones del proyecto. ${ }^{61}$ En coherencia con esa intención inicial del relator, en su quinto y último informe, entre otras modificaciones en relación con el texto aprobado en primera lectura, el relator vuelve a incluir un artículo, el 20, sobre la "relación con otros convenios y acuerdos internacionales". ${ }^{2}$ Sin embargo, fue desechado en la versión final del proyecto de artículos.

Por otra parte, la República de Corea había hecho una propuesta sobre el establecimiento de un mecanismo de solución de controversias, que el relator sugirió que fuese considerado por una hipotética futura conferencia negociadora de una convención, que sería la que establecería también el preámbulo y las cláusulas finales de la misma. ${ }^{63}$

Tras un periodo relativamente corto de trabajo — sobre todo en comparación con los trabajos de la Convención de Nueva York de 1997, que se habían extendido durante 24 años-, la CDI adoptó en segunda lectura en su sexagésimo periodo de sesiones en 2008 un preámbulo y 19 proyectos de artículos, así como comentarios a todos ellos. ${ }^{64}$ Durante el desarrollo de estos trabajos, la CDI contó con el asesoramiento de expertos del Programa Hidrológico International (PHI) de la Unesco y de otras organizaciones como la FAO, la UNECE o la Asociación Internacional de Hidrogeólogos (AIH), que facilitó enormemente la elaboración de un texto científicamente preciso. ${ }^{65}$

El ámbito de aplicación del proyecto de artículos abarca, a grandes rasgos, a toda clase de acuíferos transfronterizos y no solo a las aguas

\footnotetext{
61 Ibíd.

62 Ibíd., párr. 38.

63 Ibíd. párr. 41.

64 Documentos oficiales de la Asamblea General. Sexagésimo periodo de sesiones. Suplemento no. 10, A/63/10, 2008.

65 Raya Marina Stephan, "The Draft Articles on the Law of Transboundary Aquifers: the process at the UN ILC," International Community Law Review 13, no. 3 (2011): 223-235.
} 
subterráneas conectadas con otras superficiales, como ocurría en la Convención de Nueva York de 1997. Recoge, además, en líneas generales, las normas consuetudinarias básicas en relación con los recursos hídricos superficiales transfronterizos ya codificadas en aquella convención —utilización equitativa y razonable, obligación de no causar daños sensibles, obligación general de cooperar, intercambio regular de datos e información, y normas relativas a la protección, preservación y gestión-, ${ }^{66}$ ajustándolas a las características particulares de los acuíferos. Además, incorpora disposiciones especialmente diseñadas para la regulación de los acuíferos como la controvertida referencia, dentro de los principios generales, a la soberanía de los Estados del acuífero sobre la parte de estos ubicada en su territorio. ${ }^{67}$ Se hace también un especial hincapié en la protección de las zonas de recarga y descarga de los acuíferos, ${ }^{68}$ así como en la necesidad de una gestión conjunta por parte de los Estados que comparten este recurso. ${ }^{69}$

\subsection{Las discusiones ante la Asamblea General (2008-2016)}

A continuación, la Asamblea General, siguiendo las indicaciones de la CDI, adoptó durante su sexagésimo tercer periodo de sesiones la Resolución 63/124 sobre el derecho de los acuíferos transfronterizos, que anexaba el texto del proyecto de artículos. Al mismo tiempo, alentaba a los Estados interesados a que concertasen los correspondientes arreglos bilaterales o regionales para la adecuada gestión de sus acuíferos transfronterizos teniendo en cuenta sus disposiciones e incluyó el tema en el programa de su sexagésimo sexto periodo de sesiones, para examinar, entre otras cuestiones, la forma final que podría adoptar. ${ }^{70}$ Desde entonces, se ha ido postergando la decisión sobre esa forma final.

En octubre y noviembre de 2011, la Sexta Comisión consideró el tema en dos reuniones sirviéndose de un informe del Secretario General que contenía los comentarios y observaciones de los gobiernos sobre el proyecto de artículos, que seguían siendo diversos en cuanto a cuál

\footnotetext{
66 Artículos 4 y 5, 6, 7, 8 y 10 a 15 , respectivamente.

67 Artículo 3.

68 Artículo 11.

69 Artículos 7.2, 9 y 14.

70 Resolución 63/124 de la Asamblea General, de 11 de diciembre de 2008, A/RES/63/124.
} 
debería ser su forma final. Algunos Estados se mostraron partidarios de la elaboración de una convención como Portugal, Chile y El Salvador, mientras que para otros sería todavía demasiado prematuro - Austria, Colombia y México-; se necesitaba contar con una mayor práctica convencional por los Estados. Otros abogaron por que el proyecto de artículos adoptase la forma de una resolución o declaración no vinculante - China- Otros se mostraron partidarios de fomentar la elaboración de acuerdos bilaterales y regionales pues vaticinaban poco éxito a una hipotética convención -Panamá y Estados Unidos.

En enero de 2012 la Asamblea General adoptó una nueva resolución sobre esta cuestión, que anexaba, de nuevo, el proyecto de artículos. En ella enfatizó la necesidad de una gestión razonable y apropiada de los acuíferos transfronterizos a través de la cooperación internacional y se animaba nuevamente a los Estados a celebrar acuerdos bilaterales y regionales teniendo en cuenta las disposiciones del proyecto de artículos. Además, incluyó esta cuestión en la agenda de su sexagésimo octavo periodo de sesiones para seguir examinándola y tratar, en especial, el tema de su forma final. ${ }^{71}$

De conformidad con esa resolución, el Secretario General de las Naciones Unidas elaboró otro informe en 2013 en el que recogió, de nuevo, comentarios y observaciones de los gobiernos sobre el proyecto de artículos. Los Estados que en esta ocasión comentaron acerca de la forma final del proyecto lo volvieron a hacer de forma dispar: ${ }^{72}$ algunos se mostraron claramente favorables a la elaboración de una convención como Finlandia, Portugal y Kuwait; otros adoptaron posturas flexibles que no rechazaban su elaboración, mientras abogaban también por la cooperación bilateral — caso de El Salvador y Japón-, y Estados Unidos volvió a manifestar su preferencia por acuerdos específicos para cada contexto.

Siguiendo este documento, la Asamblea General formuló otra resolución anexando de nuevo el proyecto de artículos a fin de que le sirviese a los Estados de orientación en sus acuerdos y arreglos bilaterales o regionales al respecto y alentó al PHI de la Unesco a seguir prestando su asistencia científica y técnica a los Estados interesados. ${ }^{73}$ Las mismas

\footnotetext{
71 Resolución 66/104 de la Asamblea General del 13 de enero de 2012, A/RES/66/104.

72 El derecho de los acuíferos transfronterizos. Informe del Secretario General, 22 de julio de 2013, A/68/172.

73 Resolución 68/118 de la Asamblea General del 16 de diciembre de 2013.
} 
recomendaciones se hacen en la resolución de la Asamblea General de 2016 sobre este tema, al mismo tiempo que se decide incluirlo de nuevo en el programa provisional de su septuagésimo periodo de sesiones (2019). ${ }^{74}$

Puede apreciarse, por lo tanto, un cambio en el lenguaje de las resoluciones de la Asamblea General en este tema que parece otorgarle una mayor autoridad al proyecto de artículos. Se ha pasado así de animar a los Estados a tenerlos en cuenta, a que los utilicen como orientación. Ello parece sugerir tanto un reconocimiento más sólido del proyecto de artículos por parte de la comunidad internacional, como una invitación más fuerte a los Estados para que sigan las normas previstas en él. ${ }^{75}$

La idea de elaborar una convención no se ha descartado totalmente si bien no parece existir una intención de llevarla a cabo a corto plazo. Parece estarse esperando ver la aceptación por parte de los Estados del proyecto de artículos como un documento guía no vinculante, que es posible; si su utilización resulta exitosa, se pueda llegar a negociar una convención. ${ }^{76}$

\section{Reflexiones en torno a los posibles efectos jurídicos y prácticos del Proyecto de Artículos sobre el Derecho de los Acuíferos Transfronterizos}

La génesis y el estado actual del Proyecto de Artículos sobre el Derecho de los Acuíferos Transfronterizos guardan importantes similitudes — salvadas, de nuevo, las diferencias de las cuestiones reguladas y su relevancia general dentro del derecho internacional - en lo relativo a la responsabilidad del Estado por hechos ilícitos. Especialmente en cuanto a la progresiva disipación de la idea de elaborar una convención y, estrechamente relacionado con ello, con la decisión de no incluir en el proyecto de artículos una cláusula sobre resolución de conflictos.

\footnotetext{
74 Resolución 71/150 de la Asamblea General del 13 de diciembre de 2016.

75 Gabrial Eckstein y Francesco Sindico, "The law of transboundary aquifers: many ways of going forward, but only one way of standing still," Review of European, Comparative \& International Environmental Law 23, no. 1 (2014): 35.

76 En este sentido, Nele Matz-Lück, "The Benefits of Positivism: The ILC's Contribution to the Peaceful Sharing of Transboundary Groundwater," en Peace through international law: the role of the International Law Commission: a colloquium at the occasion of its sixtieth anniversary editado por Georg Nolte, (Dordrecht: Springer, 2009), 145.
} 
De los debates a propósito del Proyecto de Artículos sobre el Derecho de los Acuíferos Transfronterizos, tanto en la CDI como posteriormente en el seno de la Asamblea General y su Sexta Comisión, se desprende que, aunque no se está ante un texto perfecto, la gran mayoría de los Estados se han mostrado favorables a este. Al contrario, las discrepancias parecen surgir sobre todo en torno a cuál debe ser su forma final, de modo que esa decisión se sigue posponiendo. En todo caso, además de los argumentos esgrimidos por los Estados en el procedimiento de tramitación de este proyecto de artículos, probablemente una de las razones que con más fuerza aconsejaría el mantenimiento del status quo de este texto como proyecto de artículos para ser utilizado como guía por los Estados lo constituiría la experiencia de la reticencia de los Estados a la ratificación de la Convención de Nueva York de 1997. Además, en caso de adoptarse, esta se enfrentaría a desafíos como su relación con esa convención ${ }^{77}$ y los acuerdos preexistentes sobre acuíferos transfronterizos específicos, o con la necesidad de establecer un mecanismo de solución de controversias, del que ahora mismo carece. Una convención sin una clausula sobre resolución de conflictos tiene poca utilidad, pero, al mismo tiempo, este tipo de disposiciones resultan muy controvertidas, cuestión muy discutida en la propia Convención de Nueva York de $1997^{78}$ y en otros instrumentos jurídicos internacionales sobre recursos hídricos transfronterizos como el Acuerdo sobre el Acuífero Guaraní. ${ }^{79}$

77 Véase, entre otros: Owen McIntyre, "International water resources law and the International Law Commission Draft Articles on Transboundary Aquifers: A missed opportunity for cross- fertilization?”, International Community Law Review 13, no. 3 (2011): 237-254; Christine Traversi, "The inadequacies of the 1997 Convention on International Water Courses and 2008 Draft Articles on the Law of Transboundary Aquifers," Houston Journal of International Law 33, no. 2 (2011): 453-488, o Francesco Sindico y Laura Movilla, "The interplay between the UN Watercourses Convention and the Law on Transboundary Aquifers (Article 2)," en Convention on the Law of the Non-Navigational Uses of International Watercourses. A Commentary editado por Laurence Boisson de Chazournes, Makane Moise Mbengue, Mara Tignino, et al., (Oxford University Press, 2018).

78 Salman M. A., "The United Nations Watercourses Convention Ten Years Later: Why Has its Entry into Force Proven Difficult?”, Water International 32, no. 1 (2007): 11.

79 Francesco Sindico, "The Guarani Aquifer System and the International Law of Transboundary Aquifers," International Community Law Review 13, no. 3 (2011): 259; Francesco Sindico, Ricardo Hirata, Ricardo \& y Alberto Manganelli, “The Guarani Aquifer System: From a Beacon of Hope to a Question Mark in the Management/Governance of Transboundary Aquifers," Journal of Hydrology: Regional Studies 20, (2018): 49-59. 
Como se ha podido deducir del hecho de que muchos proyectos de artículos de la CDI que no han dado lugar a convenciones hayan sido utilizados igualmente en la práctica internacional, el éxito —en el sentido de su aplicación por los Estados y otros actores y sujetos de la sociedad internacional — de un proceso codificador no puede medirse únicamente por el resultado en forma de convención. Tampoco, en el caso que hubiese resultado en una convención, por si esta alcanza o no, con mayor o menor rapidez el número de ratificaciones necesarias para su entrada en vigor. $\mathrm{Al}$ contrario, su éxito radicaría en su aceptación práctica, sobre todo, por los Estados. Ello va a depender en gran medida de si su contenido constituye un derecho consuetudinario o - en caso de representar principalmente desarrollo progresivo en una materia, como es el caso de los proyectos de artículos sobre los acuíferos transfronterizos y los hechos ilícitos- si es capaz de consolidar o generar costumbres internacionales. ${ }^{80}$

De este modo, debe plantearse en primer lugar cuál es la naturaleza jurídica de las disposiciones del Proyecto de Artículos Relativo al Derecho de los Acuíferos Transfronterizos. Resulta difícil pronunciarse sobre el carácter consuetudinario de dichas disposiciones debido al reciente desarrollo de su regulación y la todavía escasa práctica estatal en esta materia. Este recoge algunas normas sobre las que existe un amplio consenso sobre su carácter consuetudinario en el ordenamiento jurídico internacional en general como es la prohibición de causar daños sensibles; ${ }^{81}$ también, otras que gozan de gran aceptación en el ámbito específico de las aguas superficiales, relacionadas con los que se han consolidado históricamente. Se trata de la utilización equitativa y razonable, la obligación general de cooperar, la notificación de medidas proyectadas y el intercambio de datos e información. Esta última en concreto sería la única sobre la que se podría quizás afirmar su carácter consuetudinario en relación con los acuíferos, por encontrarse presente en los escasos acuerdos sobre los

\footnotetext{
80 Véanse, entre otros, Lusa Bordin, "Reflections of...," 560.

81 Véanse en este sentido el Asunto del Canal de Corfú (Gran Bretaña c. República Popular de Albania. Sentencia del 25 de marzo de 1948. CIJ Recueil 1948, p. 15), el arbitraje sobre el Caso Lanoux (Lake Lanoux Arbitration (Spain vs. France). November 16, 1957) o el relativo a la fundición Trail (Laudos de 16 de abril de 1939 y 11 de marzo de 1941, un: Reports of International Arbitral Awards, Vol. III, 1905 y ss.).
} 
acuíferos transfronterizos en la Convención de Nueva York de 1997 y en el proyecto de artículos. ${ }^{82}$

En cuanto al resto de normas contenidas en el proyecto de artículos relativas, entre otras, a la protección de los acuíferos frente a la contaminación de los ecosistemas relacionados o de sus zonas de recarga y descarga o a su gestión conjunta, integrada y basada en el criterio de precaución, aunque se trata en opinión de los autores de normas básicas necesarias para la adecuada protección y gestión de los acuíferos teniendo en cuenta sus especiales características, no tendrían todavía la presencia, consistencia y consenso suficientes en la práctica estatal como para tildarlas de consuetudinarias. Como mucho, cabría hablar de normas emergentes. La excepción la constituiría el establecimiento de mecanismos institucionales conjuntos, que se ha hecho, aunque de forma heterogénea, por la mayoría de los escasos acuerdos de cooperación existentes sobre acuíferos transfronterizos concretos.

Si se concluye entonces que el proyecto de artículos contiene en su mayor parte disposiciones que constituyen un desarrollo progresivo, este puede llegar a tener importantes efectos jurídicos si se considera que está colmando de manera importante una laguna del ordenamiento jurídico y llega a ser suficientemente aceptado y utilizado por los Estados de manera extensa, representativa y consistente.

La autoridad del proyecto de artículos puede verse fortalecida por el escaso desarrollo del derecho internacional en esta materia, que puede favorecer su aceptación por los Estados, a falta de otro marco jurídico. ${ }^{83}$ Habrá que esperar a ver si tiene un efecto constitutivo o generador similar al que puede tener una disposición de un tratado que no representa derecho consuetudinario en el momento de su redacción, pero que consigue posteriormente esa categoría si la práctica ulterior es lo suficientemente

82 En este sentido, Eckstein, Gabriel, "Managing buried treasure across frontiers: the international Law of Transboundary Aquifers," Water International 36, no. 5 (2011): 577579 .

Según la Conclusión 11.2 de la CDI sobre la identificación del derecho consuetudinario, el hecho de que una cantidad de tratados establezcan una norma, puede indicar, pero no necesariamente, que esa norma refleja derecho consuetudinario. En este caso, el hecho de que la contengan la mayoría de los pocos tratados existentes en la materia, le otorga especial carácter indicativo. Resolución 73/203...

83 Véase, en este mismo sentido, Villalpando, “Codification Light...", 120. 
constante y uniforme. ${ }^{84}$ De este modo, tendrá que verse en qué grado los Estados acogen sus normas y principios como prácticas y las aceptan como el marco jurídico en la elaboración de acuerdos bilaterales y regionales sobre acuíferos específicos. ${ }^{85}$

En este sentido, el proyecto de artículos ha tenido ya cierta repercusión en otros instrumentos jurídicos. La Resolución 63/124 de la Asamblea General, que lo anexó por primera vez, se ha citado así en el preámbulo de la mayoría de los contados acuerdos para la gestión de acuíferos transfronterizos que se han adoptado con posterioridad a la misma: el Acuerdo sobre el Acuífero Guaraní de 2010; ${ }^{86}$ el Memorando de entendimiento para el establecimiento de un mecanismo consultivo para la gestión integrada de los recursos hídricos del Sistema Acuífero Iullemeden, Taoudeni/Tanezrouft de $2014,{ }^{87}$ y el Memorando de Entendimiento sobre el acuífero Ocotepeque-Citalá adoptado el 22 de febrero de $2019 .{ }^{88}$ Estos tres acuerdos y los otros dos adoptados con posterioridad a 2008 sobre el Acuífero Al Sag-Al Disi en $2015^{89}$ y el Sistema Acuífero Transfronterizo Stampriet en $2017^{90}$ son heterogéneos en cuanto a su forma jurídica, extensión y contenido. Sin embargo, el contenido común a todos ellos, y al proyecto de artículos de la CDI, lo constituyen al menos

84 Conclusión 11.1. c de la CDI sobre la identificación del derecho consuetudinario internacional, Resolución 73/203...

85 Eckstein y Sindico “The law of...”, 42.

86 Acuerdo sobre el Acuífero Guaraní, suscripto por la República Argentina, la República Federal de Brasil, la República del Paraguay y la República Oriental de Uruguay en San Juan, República Argentina, el 2 de agosto de 2010.

87 Memorandum of Understanding for the Establishment of a Consultation Mechanism for the Integrated Management of the Water Resources of the Iullemeden, Taoudeni/ Tanearouft Aquifer Systems, Algeria, Benin, Burkina Faso, Mali, Mauritania, Niger, Nigeria, $2^{\text {nd }}$ Council of Ministers of Gicresait Project, Abuja, Nigeria, 28 March 2014.

88 Memorando de entendimiento entre El Salvador y Honduras, adoptado en el marco del Proyecto Governance of Groundwater Resources in Transboundary Aquifers (GGRETA), financiado por la Agencia Suiza para el Desarrollo y la Cooperación (COSUDE).

89 Agreement between Jordan and Saudi Arabia for the Management and Utilization of the Groundwaters in the Al Sag-Al Disi Aquifer, Riyadh, 30 April 2015.

90 Mecanismo de cooperación multipaís sobre el Sistema Acuífero Stampriet entre Bostusana, Namibia y Sudáfrica, dentro de la estructura de la Orasecom (Decision of the Council of the Orasecom, 34th ordinary meeting, Windhoek, Namibia, 17-18 August 2017; and Orsecom Forum of the Parties, ordinary meeting, Kasane, Botswana, 16 November 2017). 
la obligación de intercambio de datos e información y el establecimiento de un mecanismo institucional cuyas funciones son como mínimo ese intercambio.

El proyecto de artículos se menciona también en las Disposiciones Modelo sobre Aguas Subterráneas Transfronterizas de 2012 de la UNECE, tanto en su introducción —en la que se refiere al proyecto de artículos como el "desarrollo más relevante y reciente" y una "consolidación de los principios generales del Derecho Internacional del agua aplicables" como en muchos de los comentarios a estas.

En el terreno jurisprudencial, no consta todavía una referencia al proyecto de artículos en el marco de la Corte Internacional de Justicia u otro organismo jurisdiccional internacional. No obstante, se encuentra, en el ámbito nacional, al menos una decisión de la Sala Constitucional de la Corte Suprema de Justicia de Costa Rica del año 2010 que se refiere al artículo 6 -obligación de no causar un daño sensible - del proyecto de artículos en el contexto de una disputa sobre un proyecto minero. ${ }^{92}$

\section{Conclusiones}

No obstante la enorme importancia de los acuíferos transfronterizos, su régimen jurídico internacional se encuentra todavía poco desarrollado, lo que constituye al proyecto de artículos de la CDI sobre el derecho de los acuíferos transfronterizos en uno de sus desarrollos de más autoridad y detalle a nivel global.

El estudio llevado a cabo permite apreciar cómo la aplicación práctica de un proyecto de artículos de la CDI no depende tanto de su forma final, sino de su aceptación práctica, dada por el valor jurídico de su contenido. Es decir, de su naturaleza consuetudinaria o de desarrollo progresivo y, en este último caso, de su capacidad para consolidar o generar nuevas costumbres internacionales. En concreto, el proyecto de artículos sobre los acuíferos transfronterizos contiene normas claramente consuetudinarias en el derecho internacional general como la prohibición

\footnotetext{
91 ECE/MP.WAT/2012/L.5, Appendix, párr. 3.

92 Decisión con fecha del 16 de abril 2010, voto no. 10-006922 de la Sala Constitucional, párrafo XIII.

Véase al respeto: Nicolás Boeglin, "Acuíferos transfronterizos: respuestas desde el derecho internacional y vacíos en Centroamérica", Boletín Geológico y Minero 23, no. 3 (2012): 240-241.
} 
de causar daños sensibles, otras que pueden considerarse como tales en el ámbito de la gestión de los recursos hídricos internacionales y otras que, en mayor o menor medida, pueden caracterizarse como emergentes, como el establecimiento de órganos conjuntos para su gestión.

Salvadas las diferencias del interés general para el derecho internacional de cada uno de los temas, lo ocurrido, entre otros, con los trabajos de la CDI sobre la responsabilidad de los Estados, especialmente con el proyecto de artículos sobre hechos ilícitos, puede servir de ejemplo para anticipar los posibles efectos jurídicos que puede tener el relativo al derecho de los acuíferos transfronterizos, aun cuando no llegue a convertirse en una convención. De hecho, aunque todavía tímida, ya puede apreciarse cierta aplicación práctica del mismo, que puede verse favorecida, sobre todo, por el hecho de que este ámbito, relativamente reciente del ordenamiento jurídico internacional, se está desarrollando a través de acuerdos bilaterales y multilaterales sobre acuíferos específicos. Estos acuerdos, que, previsiblemente, seguirán aumentando en los próximos años, reflejan ya en muchos casos el contenido de ese proyecto de artículos y, en especial, la obligación de intercambio de datos e información y el establecimiento de mecanismos institucionales conjuntos.

\section{Referencias}

Boeglin, Nicolás. "Acuíferos transfronterizos: respuestas desde el derecho internacional y vacíos en Centroamérica". Boletín Geológico y Minero 23, no. 3 (2012): 235-48.

Burchi, Stefano. "Legal frameworks for the governance of international transboundary aquifers: Pre- and post-ISARM experience." Journal of Hidrology: Regional Studies 20, (2018): 15-20.

Caron, David D. "The ILC Articles on State Responsibility: The Paradoxical Relationship between Form and Authority." The American Journal of International Law 96, (2002): 856-73.

Crawford, James \& Olleson, Simon, "The Continuing Debate on a UN Convention on State Responsibility." International and Comparative Law Quarterly 54, no. 4 (2005): 959-71.

Crawford, James. The International Law Commission's articles on state responsibility: introduction, text, and commentaries. Cambridge University Press, 2002.

D’Aspremont, Jean. "The Politics of Deformalization in International Law." Goettingen Journal of International Law 3, no. 2 (2011): 503-50. 
D'Aspremont, Jean. Formalism and the sources of International Law. A Theory of the Ascertainment of Legal Rules. Oxford University Press, 2011.

Eckstein, Gabriel \& Sindico, Francesco. "The law of transboundary aquifers: many ways of going forward, but only one way of standing still." Review of European, Comparative \& International Environmental Law 23, no. 1 (2014): 32-42.

Eckstein, Gabriel. "Managing buried treasure across frontiers: the international Law of Transboundary Aquifers." Water International 36, no. 5 (2011): 573-83.

López Martín, Ana Gemma. "La codificación del Derecho Internacional en el umbral del Siglo XXI. Luces y sombras en la labor de la CDI". Anuario Hispano-Luso-Americano de Derecho Internacional 15, (2001): 367-90.

Lusa Bordin, Fernando. "Reflections of Customary International Law: The Authority of Codification Conventions and ILC Draft Articles in International Law." International and Comparative Law Quarterly 63, no. 3 (2014): 535-67. https://doi.org/10.1017/S0020589314000220

Matz-Lück, Nele. "The Benefits of Positivism: The ILC's Contribution to the Peaceful Sharing of Transboundary Groundwater." En Peace through international law: the role of the International Law Commission: a colloquium at the occasion of its sixtieth anniversary editado por Georg Nolte, 125-50. Dordrecht: Springer, 2009.

McIntyre, Owen. "International water resources law and the International Law Commission Draft Articles on Transboundary Aquifers: A missed opportunity for cross- fertilization?" International Community Law Review 13, no. 3 (2011): 237-54.

Mechlem, Kerstin. "Moving Ahead in Protecting Freshwater Resources: The International Law Commission's Draft Articles on Transboundary Aquifers." Leiden Journal of International Law 22, (2009): 801-21.

Movilla Pateiro, Laura, "Ad hoc Legal Mechanisms Governing Transboundary Aquifers: Current Status and Future Prospects." Water International 40, no. 6 (2016): 851-65.

OMM/Unesco, Glosario Hidrológico Internacional, OMm no. 385, 2012.

Pastor Ridruejo, José Antonio "Las Naciones Unidas y la codificación del Derecho Internacional: aspectos jurídico-políticos”. En Las Naciones Unidas y el Derecho Internacional coordinado por Carlos Fernández de Casadevante y Francisco Javier Quel López, 173-87. Barcelona: Ariel, 1997. 
Rey Caro, Ernesto José. "Daño transfronterizo ambiental y asignación de la pérdida". Tutela Jurídica del Medio Ambiente, (2008): 85-92.

Richey, Alexandra S., Thomas, Brian F., Lo, Min-Hui, et al. "Quantifying Renewable Groundwater Stress with GRACE.” Water Resources Research 51, no. 7 (2015): 5217-38.

Salman M. A., Salman. "The United Nations Watercourses Convention Ten Years Later: Why Has its Entry into Force Proven Difficult?" Water International 32, no. 1 (2007): 1-15.

Sindico, Francesco. "The Guarani Aquifer System and the International Law of Transboundary Aquifers". International Community Law Review 13, no. 3 (2011): 255-72.

Sindico, Francesco, Hirata, Ricardo, \& Manganelli, Alberto. "The Guarani Aquifer System: From a Beacon of hope to a Question Mark in the Management/Governance of Transboundary Aquifers." Journal of Hydrology: Regional Studies 20, (2018): 49-59.

Sindico, Francesco \& Movilla, Laura. "The interplay between the UN Watercourses Convention and the Law on Transboundary Aquifers (Article 2)." En Convention on the Law of the Non-Navigational Uses of International Watercourses. A Commentary, editado por Laurence Boisson de Chazournes, Makane Moise Mbengue, Mara Tignino, et al. Oxford University Press, 2018.

Stephan, Raya Marina. "The Draft Articles on the Law of Transboundary Aquifers: the process at the UN ILC." International Community Law Review 13, no. 3 (2011): 223-35.

Traversi, Christine. "The Inadequacies of the 1997 Convention on International Water Courses and 2008 Draft Articles on the Law of Transboundary Aquifers." Houston Journal of International Law 33, no. 2 (2011): 453-88.

Villalpando, Santiago. "Codification Light: A New Trend in the Codification of International Law at the United Nations." Anuário Brasileiro de Direito Internacional 8, no. 2 (2013): 117-55.

Villar, Pilar Carolina. "International Cooperation on Transboundary Aquifers in South America and the Guarani Aquifer Case." Revista Brasileña de Política Internacional 59, no. 1 (2016): 1-20.

World Water Assessment Programme. Water, a Shared Responsibility. The United Nations World Water Development Report 2006. Unesco, 2006.

World Water Assessment Programme. Water for a Sustainable World, The UN World Water Development Report 2015. Unesco, 2015. 